\title{
RNA sequencing-based identification of potential targets in acute myeloid leukemia: A case report
}

\author{
OMAR S. EL-MASRY ${ }^{1}$, ALI M.AL-AMRI ${ }^{2}$, AHLAM ALQATARI $^{3}$ and KHALDOON ALSAMMAN ${ }^{1}$ \\ ${ }^{1}$ Department of Clinical Laboratory Sciences, College of Applied Medical Sciences, Imam Abdulrahman Bin Faisal University, \\ Dammam, Eastern Province 34212; ${ }^{2}$ Department of Internal Medicine/Oncology; ${ }^{3}$ Hematology Laboratory/Hematopathology, \\ College of Medicine, Imam Abdulrahman Bin Faisal University, King Fahd Hospital of The University, \\ Al-Khobar, Eastern Province 34445, Kingdom of Saudi Arabia
}

Received April 30, 2020; Accepted August 6, 2020

DOI: $10.3892 /$ br.2020.1349

\begin{abstract}
Acute myeloid leukemia (AML) refers to heterogenous types of blood cancer which possess a complicated genomic landscape, and multiple novel mutational alterations are frequently being reported. Herein, a case report of a 37-year old AML patient is presented, who was diagnosed following laboratory investigation after admission. The patient had thrombocytopenia, and three consecutive blast counts of 40,30 and $41 \%$, respectively. A blood sample was collected for whole-genome RNA sequencing to understand the transcriptomic profile at the time of diagnosis and compared with a matched female control. Gene expression was quantified using the RSEM software package. Bioinformatics analysis revealed a significant number of differentially expressed genes in the patient, suggesting a marked change in the transcriptomic landscape in this patient. By mining the bioinformatics data and screening the highly expressed genes with $\geq 80 \%$ probability of gene expression, four novel genes were highlighted that may serve as potential future targets in AML patients; $\mathrm{Rh}$ associated glycoprotein, succinate receptor 1, transmembrane-4 L-six family member-1 and ADGRA3, although further validation of their value is required.
\end{abstract}

\section{Introduction}

Acute myeloid leukemia (AML) is a major subtype of leukemia resulting from the uncontrolled proliferation and incomplete

Correspondence to: Dr Khaldoon Alsamman or Dr Omar S. El-Masry, Department of Clinical Laboratory Sciences, College of Applied Medical Sciences, Imam Abdulrahman Bin Faisal University, King Faisal Road, Rakkah, Dammam, Eastern Province 34212, Kingdom of Saudi Arabia

E-mail:kmalsamman@iau.edu.sa

E-mail: oselmasry@iau.edu.sa

Key words: acute myeloid leukemia, RNA sequencing, ADGRA3, $\mathrm{Rh}$ associated glycoprotein, succinate receptor 1 , transmembrane- 4 L-six family member-1 differentiation of the myeloid progenitor in the bone marrow with possible spread of myeloblasts to the blood, liver or spleen (1). According to the World Health Organization (WHO), AML is diagnosed following $\geq 20 \%$ myeloblasts in the blood or bone marrow smears, the characteristics of which is of crucial importance in the classification of the disease $(2,3)$. The European Leukemia Net (ELN) has updated their classification guidelines to match those of the WHO to assist in consistent diagnosis, management and monitoring (4). Of note, the genomic landscape of AML is complicated resulting from different classes of genetic mutations (5). In addition, it has been reported that acquisition of additional mutations as a consequence of genomic instability may contribute to the incidence of AML, reviewed in (5). In 50\% of AML patients, a normal karyotype is observed; however, advanced sequencing techniques have assisted in identifying novel genetic alterations that contribute to the progression of AML (6-8). In this respect, RNA sequencing technology has been used to examine the transcription rates of both wildtype and mutated alleles to assess allelic imbalances in patients with AML (9). It has been reported that $99 \%$ of mutated DNA is transcribed into RNA in animal model tumor cells (10). This suggests that RNA transcripts reflect the genotype of tumor cells and may assist in understanding the genetic landscape of various types of cancer, including AML, and as such, is becoming an important factor with regards to personalized medicine and clinical decision making.

\section{Case report}

The present study was reviewed and approved by the Institutional Review Board of Imam Abdulrahman Bin Faisal University (approval no. IRB \#2017-03-147) and patient consent was obtained prior to participation.

A 37 year old female presented with general weakness, bone pain and Petechiae, suggestive of the thrombocytopenia (Table I) for 2 weeks. The patient suffered from photosensitivity, arthralgia and solar urticaria for the last 6 years, and was followed up by the Dermatology Clinic. She also had a nephew who was diagnosed with acute myeloid leukemia at the age of 6. Clinical examination showed a well-developed, well-nourished and alert female. There was no evidence of lymphadenopathy or organomegaly. She had scattered 
Table I. Complete and differential blood counts.

\begin{tabular}{lccc}
\hline Parameter (reference range) & 1st check & 2nd check & 3rd check \\
\hline Red blood cells (4.2-5.5), Mil/ul & 4.51 & 3.9 & 4.14 \\
Hemoglobin (12-16), g/dl & 11.5 & 10 & 10.6 \\
Hematocrit (37-47), \% & 35 & 29.9 & 31.5 \\
Red blood cell distribution width (11.5-14.5) & 17.7 & 18.1 & 17.6 \\
Mean corpuscular volume (80-94), fl & 77.6 & 76.7 & 76.1 \\
Mean corpuscular hemoglobin (27-32), pg & 25.6 & 25.6 & 25.5 \\
Mean corpuscular hemoglobin concentration (32-36), g/dl & 33 & 33.4 & 33.5 \\
White blood cells (4-11), k/ul & 8.6 & 7.9 & 9.1 \\
Corrected white blood cell (4-11), k/ul & 8.4 & - & - \\
Platelet (140-450), k/ul & 62 & 45 & 47 \\
Mean platelet volume (7.2-11.1), fl & 8.4 & 8.8 & 22 \\
Segmented (38-79), $\%$ & 15 & 23 & 2 \\
Band lymphocytes (0-3), $\%$ & 8 & 2 & 22 \\
Lymphocytes (12-15), & 23 & 23 & 4 \\
Monocytes (0-10), $\%$ & 2 & - & - \\
Atypical lymphocytes (0), $\%$ & 9 & - & - \\
Metamyelocytes (0), \% & 1 & 3 & 2 \\
Myelocytes (0), $\%$ & 1 & 3 & 1 \\
Promyelocytes (0), $\%$ & 1 & - & - \\
Eosinophils (0-8), $\%$ & - & 2 & 6 \\
Basophiles (0-1), $\%$ & - & 5 & 41 \\
Blasts (0), $\%$ & 40 & - & \\
Nucleated red blood cells (0) & 2 & 30 & \\
\hline
\end{tabular}

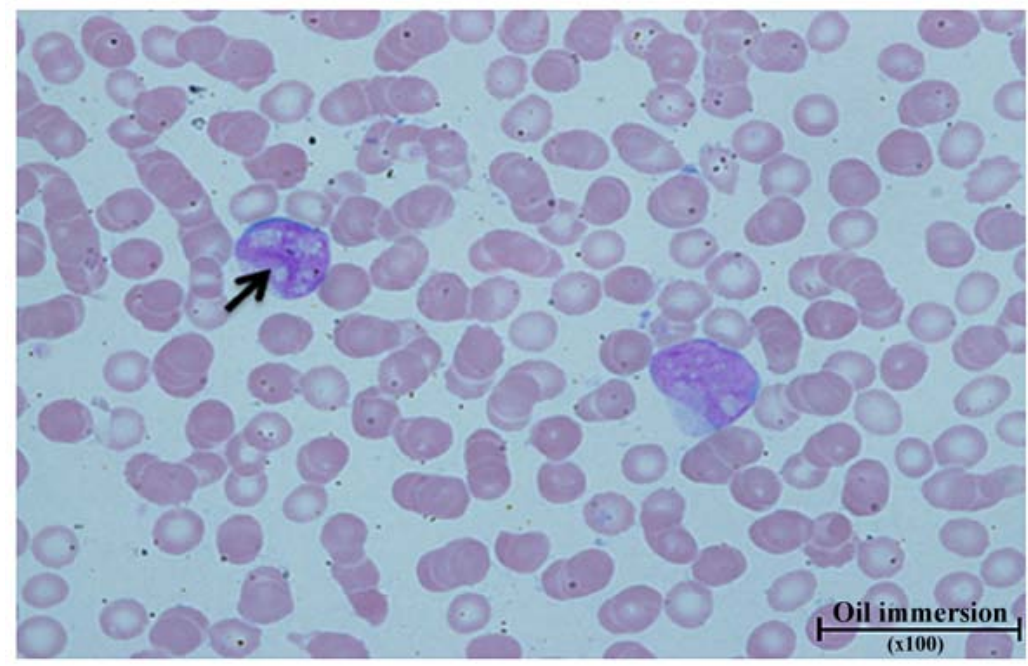

Figure 1. Peripheral blood smear showing large myeloblasts with a high nucleus to cytoplasm ratio. A nucleus with cribriform pattern, open chromatin (black arrow) and multiple nucleoli are shown. Magnification, x100, oil immersion. Scale bar, $45 \mu \mathrm{m}$.

Petechiae, but no purpura or ecchymosis. Laboratory analysis of blood smears and complete blood counts revealed significant relative leukocytosis and $>20 \%$ blast counts. Of note, according to the WHO definition, the presence of blast counts $>20 \%$ is confirmatory of an AML diagnosis (11). According to the definition of the French-American-British classification system (2), blast counts $>30 \%$ are confirmatory of a diagnosis of AML (3). Blood smears were assessed on admission along with complete blood counts, and the blast count was $40 \%$, which was confirmed in subsequent blood samples, with blasts counts of 30 and $41 \%$. Blast cells were large with a high nuclear to cytoplasmic ratio, fine opened chromatin and irregular nuclear outlines, and some cells showed inclusion of Auer-rods. Additionally, several cells showed fine chromatin with 2-3 nucleoli. Some blasts showed convoluted cribriform nuclei (Fig. 1). Morphological blood sample analysis and 
Table II. Cytogenetics report.

Test name

Results

Cytogenetics (CBFB)-FISH

Cytogenetics MLL-FISH

Chromosomal analysis neoplastic study

Cytogenetics PML/RARA-FISH

Cytogenetics RUNX1/RUNX1T1-FISH

Cytogenetics BCR/ABL1-FISH
$100 \%$ normal CBFB

$100 \%$ normal MLL

$47, \mathrm{XX},+8[18] / 46, \mathrm{XX}[1]$

100 normal PML/RARA

$88 \%$ trisomy RUNX1T1

$100 \mathrm{BCR} / \mathrm{ABL} 1$ normal
Normal

Normal

Abnormal

Normal

Abnormal

Normal

FISH, fluorescence in situ hybridization; CBFB, core-binding factor subunit $\beta$; MLL, mixed lineage leukemia; PML-PARA, promyelocytic leukemia/retinoic acid receptor $\alpha$; RUNX1, runt-related transcription factor 1; RUNX1T1, RUNX1 partner transcriptional co-repressor 1; $\mathrm{BCR}$, breakpoint cluster region protein; ABL1, tyrosine-protein kinase; BCR/ABL1, BCR-ABL fusion gene (Philadelphia chromosome).

blood counts (Table I) suggested a diagnosis of AML. The hematopathologist recommended bone marrow analysis, for karyo-and immunotyping.

The patient was then transferred to the King Fahd Specialist Hospital, wherein molecular analysis, bone marrow analysis, karyotyping and immunophenotyping were performed. The cytogenetics report (Table II) of bone marrow aspirates indicated the presence of an abnormal tumoral clone with trisomy $8(47, \mathrm{XX},+8[18] / 46, \mathrm{XX}[1])$; this karyotype was confirmed by fluorescence in situ hybridization, which showed $88 \%$ trisomy 8 (Data not shown); a karyotype abnormality that is associated with a moderate risk of AML according to ELN (12).

Post treatment bone marrow aspirates and biopsy from the right posterior iliac crest were assessed. The biopsy was a single core $(0.9 \mathrm{~cm}$ in length) in which the cellular area showed $<10 \%$ cellularity with stromal edema and serous degeneration that was deemed to be therapy-related. A mixture of lymphocytes, macrophages, plasma cells and a few megakaryocytes were observed in the cellular area. There was no increase in blast cells as indicated by the negative immunostaining of CD117. Reticulin staining showed scattered linear reticulin indicating bone marrow fibrosis grading of MF-0, which is indicative of a normal architecture (13). The bone marrow aspirates showed marked suppression of trilineage hematopoiesis with a relative increase in lymphocytes and macrophages. Differential counts showed $7 \%$ erythroid precursors, $2 \%$ blast cells, $2 \%$ segments, $86 \%$ lymphocytes and $2 \%$ plasma cells. The complete blood count, which was performed on the same day showed marked leucopenia, neutropenia and thrombocytopenia. The differential blood counts indicated that lymphocytes accounted for $91.8 \%$ of the count, monocytes $4.9 \%$ and basophils $3.3 \%$. A case of AML showing hypocellular marrow with $2 \%$ blasts was recommended for bone marrow re-evaluation. The patient then underwent Allogenic stem cell bone marrow transplantation, as recommended as a consolidation treatment for patients with trisomy 8 AML (12). Bone marrow aspirates and biopsy were assessed again 5 months later; the marrow aspirates were aparticulated and diluted, almost mimicking the peripheral blood, with no blast cells observed upon scanning. The biopsy was $1.4 \mathrm{~cm}$ in length, in which the cellular area examined showed intertrabecular hemorrhage with crushing and tissue loss. A cellularity of $10 \%$ (marked hypocellular marrow) was also reported with trilineage hematopoiesis and no blast cells were observed. Flow cytometry analysis showed $0.1 \%$ positivity for the gated population of CD45dim/CD34. The patient passed away in April 2019.

In the present case report, the transcriptomic landscape was assessed in this patient at the time of AML diagnosis by performing whole genome RNA sequencing $(14,15)$. This was followed by gene expression quantification using the RSEM software package (16). Bioinformatics analysis and the software used are described in the supplementary materials and methods. The bioinformatics data showed a marked number of differentially expressed genes when compared with a matched female control profile; $>2,500$ differentially upregulated genes ( $\log _{2}$ fold change $\geq 1$, probability of $\geq 0.8$ (where the P-value was used to calculate the probability of gene expression) and 252 downregulated genes $\left(\log _{2}\right.$ fold change $\leq-1$, probability $\geq 0.8$ ) (Fig. 2). The volcano plots (NOISeq method) (17) in Fig. 2B also shows the number of non-differentially expressed genes $\left(14,087 ; \log _{2}\right.$ fold change $<1$ and $>-1$, probability of $\left.<0.8\right)$. Genes were sorted according to the $\log _{2}$ fold change and the probability of being upregulated. Genes with a $\log _{2}$ fold change $<5$ and a probability $<80 \%$ were excluded from subsequent analysis. In this respect, $\log _{2}$ (fold change) was calculated as follows: $\log [($ gene expression in AML/gene expression in control)/2], where gene expression in AML/gene expression in control=the actual fold change. The following formula was used to convert $\log _{2}$ (fold change values) to the actual fold change: Actual fold change $=2^{\log 2 \text { (fold change) }}$. Gene ontology analysis was used during the filtration process, which resulted in identification of possible crucial hits that may be of potential importance in disease progression and in clinical management of the patient. ADGRA3, Rh associated glycoprotein (RhAG), succinate receptor 1 (SUCNR1) and transmembrane-4 L-six family member-1 (TM4SF1) are all cell membrane proteins that were found to be upregulated in this patient compared with the corresponding control, with $\log _{2}$ fold change and gene expression probability values of 5.25, 91.2\%; 7.2, 93\%; 9 , $97 \%$; and $8.6,97 \%$, respectively. Table III shows the $\log _{2}$ (fold change), the converted fold change values and the gene expression probabilities. The results of RNA sequencing were further validated by quantitative (q)PCR. The sequences of the primers are presented in Table SI. The results confirmed the significant upregulation of RHAG, SUCNR1 and TM4SF1, with 2-DDCq values of 50.6, 266.5 and 392,476.7respectively (Fig. S1). These 


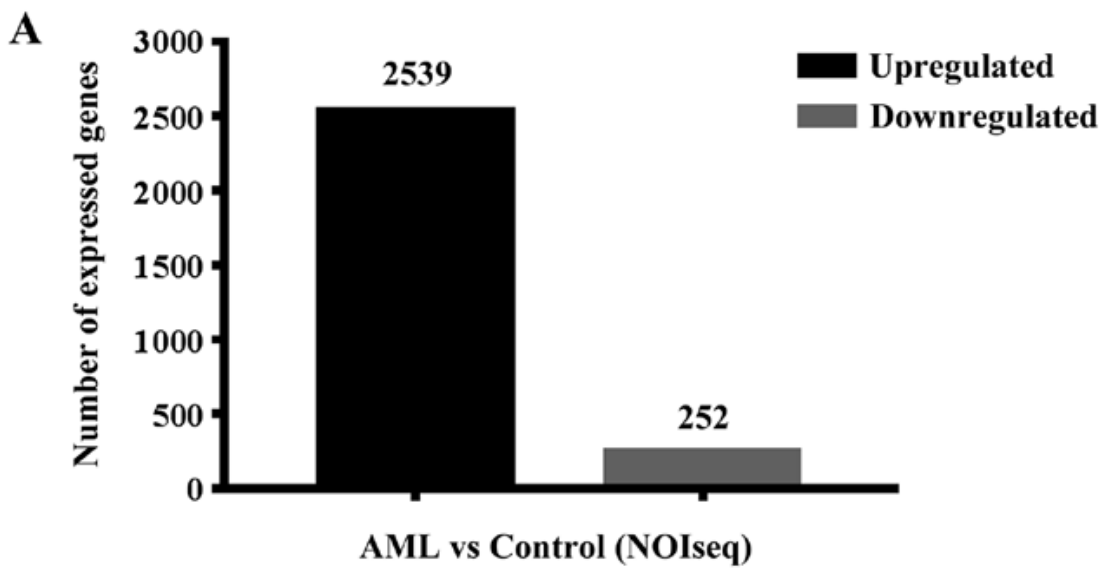

B

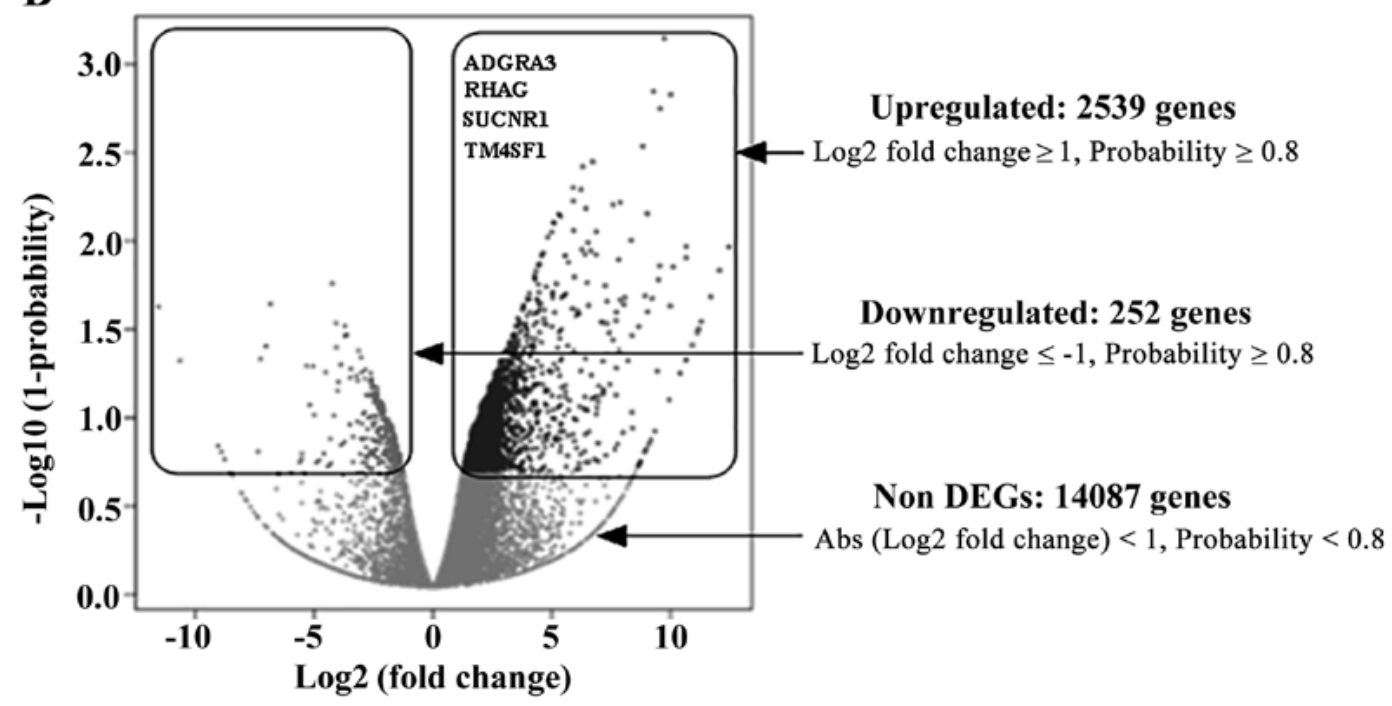

Volcano plot control vs AML. NOIseq method

Figure 2. Differentially expressed genes in the patient with AML compared with a healthy matched-control. (A) A total of 2,539 upregulated and 252 downregulated genes were identified in the patient with AML. (B) Volcano plot showing the up and downregulated genes, and the criteria used to define whether a gene was significantly dysregulated. Amongst the overexpressed genes, ADGRA3, RHAG, SUCNR1 and TM4SF1 were further considered as potential targets in AML. The $\log _{2}$ fold change and the probability of gene expression for each gene compared with the corresponding control were: ADGRA3 (5.25, 91.2\%), RHAG $(7.2,93 \%)$, SUCNR1 $(9,97 \%)$ and TM4SF1 $(8.6,97 \%)$, respectively.

values show the number of times each gene is expressed more in the AML case compared with the control sample.

To assess the genetic variations in this patient compared with the reference genome, single nucleotide polymorphism (SNP) and mutation analyses were performed. Briefly, data analyses revealed that the mapping ratio with the reference gene sequences was $75 \%$, a total of 15,763 genes were identified in this patient. Of those, 1,251 novel coding transcripts were reported for this patient. In total, 10,444 novel transcripts were generated, including the 1,251 novel gene transcripts that require further characterization, 1,160 novel non-coding transcripts and 8,033 novel splicing variants of known genes. Genome analysis toolkit (18) was used for insertion/deletion and SNP analyses. Transition events that involved an interchange of purine nucleotides [adenine (A) and guanine (G)] or pyrimidine nucleotides [cytosine $(\mathrm{C})$ and thymine $(\mathrm{T})$ ] accounted for 113,011 events in total. There were 43,161 transversion events involving the transition of purine nucleotides to pyrimidine nucleotides or vice versa. Transversion events observed included A-C, A-T, C-G and G-T. The transition and transversion events are summarized in Table SII). The results revealed intronic mutations in fms-like tyrosine kinase 3 (FLT3), mutant variants of which are present in $\sim 30 \%$ of AML cases according to a recent report (19). Another important mutation observed in the present study was in the NRAS gene, which is a common genetic signature observed in AML patients (20). In the present study, two exonic and one intronic mutation of NRAS were observed. In addition, intronic mutations were also identified in nucleophosmin 1 gene (NPM1). According to Heath et al (21), NPM1 was frequently mutated in one third of AML patients and its mutated forms were associated with improved prognosis (21). In this regard, it has been reported that mutations in AML belong to two main classes: i) class I, which consists of mutations in genes that can stimulate proliferation and cell survival (such as mutations in Ras, JAK2 and FLT3) and ii) class II, where mutations affect cell differentiation and the apoptotic machinery (such as mutations in MLL, CEBPA and NPM1). A third class of mutations was also reported, which included mutations that affect epigenetic modifications (such as mutations in ASXL1 and DNMT 3a) (5). 
Table III. Genes with potential significance in acute myeloid leukemia.

\begin{tabular}{lccc}
\hline $\begin{array}{l}\text { Gene } \\
\text { name }\end{array}$ & $\begin{array}{c}\log _{2} \\
\text { (fold change) }\end{array}$ & $\begin{array}{c}\text { Actual } \\
\text { fold change }\end{array}$ & $\begin{array}{c}\text { Probability of } \\
\text { expression, \% }\end{array}$ \\
\hline ADGRA3 & 5.25 & 38.05 & 91.2 \\
RhAG & 7.2 & 147.03 & 93 \\
SUCNR1 & 9 & 512 & 97 \\
TM4SF1 & 8.6 & 388.02 & 97 \\
\hline
\end{tabular}

RhAG, Rh associated glycoprotein; SUCNR1, succinate receptor 1; TM4SF1, transmembrane-4 L-six family member- 1 .

\section{Discussion}

AML is characterized by a heterogeneous genomic landscape resulting from numerous genetic alterations, making disease stratification and management complicated (5). RNA transcripts may assist in understanding the transcription rates of wild type and mutated genes that may serve a role in sustaining the disease process and outcomes $(9,10)$. By mining the RNA-sequencing bioinformatics data, 4 genes (ADGRA3, RHAG, SUCNR1 and TM4SF1) that were highly expressed in the patient with AML were identified. The 4 genes encoded cell surface proteins that are important in conveying extracellular signaling cascades. Thus, it was proposed that these genes may serve as potentially novel biomarkers of AML, pending further validation in additional patients, and in vitro and in vivo experimental models of AML. ADGRA3, also known as GPR125 is an adhesion G-protein-coupled membrane receptor, which mediates downstream signaling pathways by activation of $G$ proteins (22). With respect to the role of ADGRA3 in AML, it has been reported that its protein product, GPR125, is downregulated in AML, in contrast to the results of the present study, suggesting that the exact role of this gene in AML requires further study (23). It has been recently reported that upregulation of GPR 125 has prognostic value in colorectal cancer (24). In addition, the genomic data commons (GDC) data portal (25) contains 302 reports of different ADGRA3 mutations and 392 cases of copy number variations have been reported in 27 and 28 projects, respectively, indicating the role of ADGRA3 gene in neoplastic disorders of lung, bronchus, uterus, cervix, bladder, mature B cell lymphoma and others (26). SUCNR1 was significantly upregulated in the patient with AML in the present study. To the best of our knowledge, the role of SUCNR1 in AML has not yet been explored. SUCNR1 is a sensor of oxidative stress, which may assist in the cells ability to sense and manage excessive oxidative stress (27). In this regard, succinate is considered an oncometabolite, which promotes tumorigenesis by promoting angiogenesis in experimental models as well as a transgenic zebrafish cancer model (28). Furthermore, single nucleotide variants of SUCNR1 gene have been reported; however, the involvement of this gene was excluded from being implicated in the familial inheritance of gastric and rectal cancer (29). Importantly, a role of SUCNR1 in hematopoietic progenitor cell development was reported (30). The activated SUCNR1 receptor potentiates proliferation of hematopoietic precursors as well as protecting erythroleukemic cells from starvation-caused cell death (31). It has been reported that the proliferative effect of SUCNR1 in hematopoietic progenitor cells is mediated via the ERK1/2 pathway (32). In addition, 106 somatic mutations and copy number variants, gain and loss, have also been reported in multiple projects reporting these types of genotypic variants in SUCNR1 in breast, lung, bladder, uterus, ovary, liver and other types of cancer (25). Additionally, SCUNR1 overexpression was reported in ankle tissue samples in an adjuvant arthritis model (33), where arthralgia (joint pain) is a common symptom; this may reflect the universal metabolic role of SCUNR1 in multiple types of cells, including blood cells in the present study. In this regard, accumulation of succinate in multiple types of cancer has been reported, including in ovarian and thyroid cancer (34), renal and gastric carcinoma (35), and familial pheochromocytoma (36). Accumulation of succinate in cancer cells promotes oncogenesis by promoting angiogenesis via activation of STAT3 and ERK signaling through SUCNR1 $(28,37)$. However, the possibility that the upregulation of SCUNR1 in the patient presented in this case report was due to arthralgia can be excluded, as the transcriptomic profile was based on RNA transcripts extracted from blood cells, not synovial fluid samples nor the patient's chondrocytes. RhAG is an ammonia transporter, which has previously been associated with cancer based on its downregulated expression in esophageal cancer cell lines compared with normal esophageal cells (38). In contrast to the previous study, in the present study, RHAG expression was upregulated in the patient with AML, and thus may possess prognostic value as a biomarker of AML. According to the GDC data portal, 160 somatic mutations in the RHAG gene have been reported in patients from 21 different projects, suggesting a role in neoplasms of the bronchus and lung, adenocarcinomas of the colon, melanomas and other types of neoplastic diseases. Finally, the present study identified TM4SF1 as a potential crucial hit in AML. TM4SF1 is a transmembrane protein with oncogenic properties in lung cancer, where it has been shown to participate in cell migration and tumor metastasis (39). In addition, TM4SF1 was also reported to promote metastasis in pancreatic cancer (40). To the best of our knowledge the role of TM4SF1 has not yet been reported in AML.

The cell surface protein products of the identified genes found to be dysregulated may sustain the external cell signaling mechanisms in AML, and thus should be further studied to determine their diagnostic and prognostic value in patients with AML. However, the present study has some limitations. First, the expression of these dysregulated genes was not validated at the protein level. Thus, the roles of these four genes will be further verified as a continuation of the present study using in vitro and in vivo models of AML. Additionally, the differences in transcriptomic profiles were concluded from comparisons with a single healthy female control. A larger sample size of both patients and controls are required to establish more generalizable and convincing results.

In conclusion, RNA-sequencing bioinformatics data revealed the overexpression of a large number of differentially transcribed genes in this patient. Data mining led to identification of ADGRA3, RHAG, SUCNR1 and TM4SF1 as potential candidate genes for further investigation to validate their exact roles in AML. 


\section{Acknowledgements}

Not applicable.

\section{Funding}

This work was funded by the Deanship of Scientific Research at Imam Abdulrahman Bin Faisal University (grant no. 2017-099-CAMS).

\section{Availability of data and materials}

The datasets used and/or analyzed during the present study are available from the corresponding author on reasonable request.

\section{Authors' contributions}

OSEM conceived and designed the study, and wrote the manuscript. AMAA collected the clinical data and wrote the manuscript. AA collected the clinical data and revised the manuscript. KA performed the bioinformatics analysis. All authors read and approved the final manuscript.

\section{Ethics approval and consent to participate}

This study was reviewed and approved by the Institutional Review Board at Imam Abdulrahman Bin Faisal University (approval no. IRB \# 2017-03-147).

\section{Patient consent for publication}

Not applicable.

\section{Competing interests}

The authors declare that they have no competing interests.

\section{References}

1. Hasserjian RP: Acute myeloid leukemia: Advances in diagnosis and classification. Int J Lab Hematol 35: 358-366, 2013.

2. Bennett JM, Catovsky D, Daniel MT, Flandrin G, Galton DA, Gralnick HR and Sultan C: Proposals for the classification of the acute leukaemias. French-American-British (FAB) co-operative group. Br J Haematol 33: 451-458, 1976.

3. DiNardo CD, Garcia-Manero G, Pierce S, Nazha A Bueso-Ramos C, Jabbour E, Ravandi F, Cortes J and Kantarjian H: Interactions and relevance of blast percentage and treatment strategy among younger and older patients with acute myeloid leukemia (AML) and myelodysplastic syndrome (MDS). Am J Hematol 91: 227-232, 2016.

4. Döhner H, Estey E, Grimwade D, Amadori S, Appelbaum FR, Büchner T, Dombret H, Ebert BL, Fenaux P, Larson RA, et al: Diagnosis and management of AML in adults: 2017 ELN recommendations from an international expert panel. Blood 129: 424-447, 2017.

5. Lagunas-Rangel FA, Chávez-Valencia V, Gómez-Guijosa MÁ and Cortes-Penagos C: Acute myeloid leukemia-genetic alterations and their clinical prognosis. Int J Hematol Oncol Stem Cell Res 11: 328-339, 2017.

6. Martelli MP, Sportoletti P, Tiacci E, Martelli MF and Falini B: Mutational landscape of AML with normal cytogenetics: Biological and clinical implications. Blood Rev 27: 13-22, 2013.
7. Rubnitz JE, Gibson B and Smith FO: Acute myeloid leukemia. Hematol Oncol Clin North Am 24: 35-63, 2010.

8. Takahashi S: Current findings for recurring mutations in acute myeloid leukemia. J Hematol Oncol 4: 36, 2011.

9. Batcha AMN, Bamopoulos SA, Kerbs P, Kumar A, Jurinovic V, Rothenberg-Thurley M, Ksienzyk B, Philippou-Massier J, Krebs S, Blum H, et al: Allelic imbalance of recurrently mutated genes in acute myeloid leukaemia. Sci Rep 9: 11796, 2019.

10. Castle JC, Loewer M, Boegel S, Tadmor AD, Boisguerin V, de Graaf J, Paret C, Diken M, Kreiter S, Türeci O and Sahin U: Mutated tumor alleles are expressed according to their DNA frequency. Sci Rep 4: 4743, 2014.

11. Vardiman JW, Harris NL and Brunning RD: The world health organization (WHO) classification of the myeloid neoplasms. Blood 100: 2292-2302, 2002.

12. Hemsing AL, Hovland R, Tsykunova G and Reikvam H: Trisomy 8 in acute myeloid leukemia. Expert Rev Hematol 12: 947-958, 2019.

13. Thiele J, Kvasnicka HM, Facchetti F, Franco V, van der Walt J and Orazi A: European consensus on grading bone marrow fibrosis and assessment of cellularity. Haematologica 90: 1128-1132, 2005.

14. Mortazavi A, Williams BA, McCue K, Schaeffer L and Wold B: Mapping and quantifying mammalian transcriptomes by RNA-Seq. Nat Methods 5: 621-628, 2008.

15. Wang Z, Gerstein M and Snyder M: RNA-Seq: A revolutionary tool for transcriptomics. Nat Rev Genet 10: 57-63, 2009.

16. Li B and Dewey CN: RSEM: Accurate transcript quantification from RNA-Seq data with or without a reference genome. BMC Bioinformatics 12: 323, 2011.

17. Tarazona S, Furió-Tar1 P, Nueda MJ, Ferrer A and Conesa A: NOISeq: Differential expression in RNA-seq. 2013. https:// bicoductor.statistik.tu-dortmund.de/packages/3.7/bior/vignettes/ NOISwq/inst/doc/NOISeq.pdf

18. McKenna A, Hanna M, Banks E, Sivachenko A, Cibulskis K, Kernytsky A, Garimella K, Altshuler D, Gabriel S, Daly M and DePristo MA: The genome analysis toolkit: A mapreduce framework for analyzing next-generation DNA sequencing data. Genome Res 20: 1297-1303, 2010.

19. Daver N, Schlenk RF, Russell NH and Levis MJ: Targeting FLT3 mutations in AML: Review of current knowledge and evidence. Leukemia 33: 299-312, 2019.

20. Berman JN, Gerbing RB, Alonzo TA, Ho PA, Miller K, Hurwitz C, Heerema NA, Hirsch B, Raimondi SC, Lange B, et al: Prevalence and clinical implications of NRAS mutations in childhood AML: A report from the Children's oncology group. Leukemia 25: 1039-1042, 2011.

21. Heath EM, Chan SM, Minden MD, Murphy T, Shlush LI and Schimmer AD: Biological and clinical consequences of NPM1 mutations in AML. Leukemia 31: 798-807, 2017.

22. Spiess K, Bagger SO, Torz LJ, Jensen KHR, Walser AL, Kvam JM, Møgelmose ASK, Daugvilaite V, Junnila RK, Hjortø GM and Rosenkilde MM: Arrestin-independent constitutive endocytosis of GPR125/ADGRA3. Ann N Y Acad Sci 1456: 186-199, 2019.

23. Maiga A, Lemieux S, Pabst C, Lavallée VP, Bouvier M, Sauvageau $G$ and Hébert J: Transcriptome analysis of $G$ protein-coupled receptors in distinct genetic subgroups of acute myeloid leukemia: Identification of potential disease-specific targets. Blood Cancer J 6: e431, 2016.

24. Wu Y, Chen W, Gong L, Ke C, Wang H and Cai Y: Elevated G-protein receptor 125 (GPR125) expression predicts good outcomes in colorectal cancer and inhibits wnt $/ \beta$-catenin signaling pathway. Med Sci Monitor 24: 6608-6616, 2018.

25. Institute NC: Genomic data commons data portal. https://portal. gdc.cancer.gov/genes/ENSG00000152990. J 2020.

26. Institute NC: Genomic data commons data portal. https://portal. gdc.cancer.gov/genes/ENSG00000198829. J 2020.

27. Louer EMM, Lores-Motta L, Ion AM, Den Hollander AI and Deen PMT: Single nucleotide polymorphism rs13079080 is associated with differential regulation of the succinate receptor 1 (SUCNR1) gene by miRNA-4470. RNA Biol 16: 1547-1554, 2019.

28. Mu X, Zhao T, Xu C, Shi W, Geng B, Shen J, Zhang C, Pan J, Yang J, Hu S, et al: Oncometabolite succinate promotes angiogenesis by upregulating VEGF expression through GPR91-mediated STAT3 and ERK activation. Oncotarget 8: 13174-13185, 2017.

29. Thutkawkorapin J, Picelli S, Kontham V, Liu T, Nilsson D and Lindblom A: Exome sequencing in one family with gastric- and rectal cancer. BMC Genet 17: 41, 2016. 
30. Macaulay IC, Tijssen MR, Thijssen-Timmer DC, Gusnanto A, Steward M, Burns P, Langford CF, Ellis PD, Dudbridge F, Zwaginga JJ, et al: Comparative gene expression profiling of in vitro differentiated megakaryocytes and erythroblasts identifies novel activatory and inhibitory platelet membrane proteins. Blood 109: 3260-3269, 2007.

31. Ariza AC, Deen PMT and Robben JH: The succinate receptor as a novel therapeutic target for oxidative and metabolic stress-related conditions. Front Endocrinol (Lausanne) 3: 22, 2012.

32. Hakak Y, Lehmann-Bruinsma K, Phillips S, Le T, Liaw C, Connolly DT and Behan DP: The role of the GPR91 ligand succinate in hematopoiesis. J Leukoc Biol 85: 837-843, 2009.

33. Chen H, Pan T, Liu P, Wang P and Xu S: Baihu Jia Guizhi decoction improves rheumatoid arthritis inflammation by regulating succinate/SUCNR1 metabolic signaling pathway. Evid Based Complement Alternat Med 2019: 3258572, 2019.

34. Bardella C, Pollard PJ and Tomlinson I: SDH mutations in cancer. Biochim Biophys Acta 1807: 1432-1443, 2011.

35. Ricketts C, Woodward ER, Killick P, Morris MR, Astuti D, Latif $F$ and Maher ER: Germline SDHB mutations and familial renal cell carcinoma. J Natl Cancer Inst 100: 1260-1262, 2008.

36. Baysal BE, Ferrell RE, Willett-Brozick JE, Lawrence EC, Myssiorek D, Bosch A, van der Mey A, Taschner PE, Rubinstein WS, Myers EN, et al: Mutations in SDHD, a mitochondrial complex II gene, in hereditary paraganglioma. Science 287: 848-851, 2000.
37. Zhao T, Mu X and You Q: Succinate: An initiator in tumorigenesis and progression. Oncotarget 8: 53819-53828, 2017.

38. Chen BS, Xu ZX, Xu X, Cai Y, Han YL, Wang J, Xia SH, $\mathrm{Hu} \mathrm{H}$, Wei F, Wu M and Wang MR: RhCG is downregulated in oesophageal squamous cell carcinomas, but expressed in multiple squamous epithelia. Eur J Cancer 38: 1927-1936, 2002.

39. Ye L, Pu C, Tang J, Wang Y, Wang C, Qiu Z, Xiang T, Zhang Y and Peng W: Transmembrane-4 L-six family member-1 (TM4SF1) promotes non-small cell lung cancer proliferation, invasion and chemo-resistance through regulating the DDR1/Akt/ERK-mTOR axis. Respir Res 20: 106, 2019.

40. Yang JC, Zhang Y, He SJ, Li MM, Cai XL, Wang H, Xu LM and Cao J: TM4SF1 promotes metastasis of pancreatic cancer via regulating the expression of DDR1. Sci Rep 7: 45895, 2017.

This work is licensed under a Creative Commons Attribution-NonCommercial-NoDerivatives 4.0 International (CC BY-NC-ND 4.0) License. 